Pacific Journal of Mathematics

EXTREME COPOSITIVE QUADRATIC FORMS 


\title{
EXTREME COPOSITIVE QUADRATIC FORMS
}

\author{
L. D. BAUMerT
}

A real quadratic form $Q=Q\left(x_{1}, \cdots, x_{n}\right)$ is called copositive if $Q\left(x_{1}, \cdots, x_{n}\right) \geqq 0$ whenever $x_{1}, \cdots, x_{n} \geqq 0$. If we associate each quadratic form $Q=\sum q_{i j} x_{i} x_{j} \quad q_{i j}=q_{j i}(i, j=1, \cdots, n)$ with a point $\left(q_{11}, \cdots, q_{n n}, \sqrt{2} q_{12}, \cdots, \sqrt{2} q_{n-1, n}\right)$ of Euclidean $n(n+1) / 2$ space then the copositive forms constitute a closed covex cone in this space. We are concerned with the extreme points of this cone. That is, with those copositive quadratic forms $Q$ for which $Q=Q_{1}+Q_{2}$ (with $Q_{1}, Q_{2}$ copositive) implies $Q_{1}=a Q, Q_{2}=(1-a) Q, 0 \leqq a \leqq 1$. We show that if $Q\left(x_{1}, \cdots\right.$, $\left.x_{n}\right), n \geqq 2$, is an extreme copositive quadratic form then for any index $i(1 \leqq i \leqq n), Q$ has a zero $u$ with $u_{1} \cdots, u_{n} \geqq 0$ where $u_{i}>0$. Further, using this fact, we establish an extension process whereby extreme forms in $n$ variables can be used to construct extreme form in $n^{\prime}$ variables, for all $n^{\prime}>n$.

Copositive quadratic forms arise in the theory of inequalities (see the references in Diananda, [2]) and also in the study of block designs (Hall and Newman, [3]). The interested reader should also see Motzkin $[4,5]$.

In $\S 2$ we indicate some of the basic results on copositive quadratic forms and describe some normalizations which will be of use later.

2. Preliminaries. As indicated above, a real quadratic form $Q=Q\left(x_{1}, \cdots, x_{n}\right)$ is called copositive if $Q\left(x_{1}, \cdots, x_{n}\right) \geqq 0$ whenever $x_{1}, \cdots, x_{n} \geqq 0$. Thus any positive semi-definite quadratic form is copositive. Further, any quadratic form all of whose coefficients are nonnegative is clearly copositive. Thus, denoting the class of positive semi-definite quadratic forms by $S$ and the nonnegative coefficient forms by $P$, we see that any quadratic form which is expressible as a sum of elements of $P$ and $S$ is necessarily copositive (i.e., $Q \in P+S$ implies $Q$ is copositive). In fact, Diananda [2] has shown that there are no other copositive quadratic forms in $n \leqq 4$ variables. On the other hand, A. Horn [3] has constructed an extreme copositive quadratic form in 5 variables which does not belong to $P+S$. The extreme copositive quadratic forms belonging to $P+S$ have been determined by Hall and Newman [3]. Thus whenever it is convenient to do so we shall restrict attention to extreme copositive forms not belonging to $P+S$.

Suppose $Q=Q\left(x_{1}, \cdots, x_{n}\right)$ is copositive; then so is $Q^{\prime}=Q\left(p_{1} x_{1}, \cdots\right.$, $\left.p_{n} x_{n}\right)$ for any choice of $p_{i}>0(i=1, \cdots, n)$. Similarly $Q$ is an extreme form if and only if $Q^{\prime}$ is also. Hence in questions of copositivity or 
extremity we may restrict ourselves to the case $q_{i i}=1(i=1, \cdots, n)$, provided $q_{i i}>0(i=1, \cdots, n)$. As copositivity implies $q_{i i} \geqq 0(i=1$, $\cdots, n$ ) the only alternative is that $q_{i i}=0$ (some $i$ ). Here the form, if copositive, is of an extremely simple type (Lemma 2, Diananda) which often allows us to consider only those variables for which $q_{i i}>0$. That is, we can often restrict attention to a sub-form for which we can insist that $q_{i i}=1\left(i=1, \cdots, n^{\prime}<n\right)$. Relabeling of the variables is another transformation which does not effect copositivity or extremity and we shall resort to it in order to simplify the statements of results.

In many instances the concept of extremity is hard to handleand it often suffices to replace it with the weaker property which (following Diananda) we call $A^{*}(n)$.

DeFinition 2.1. A quadratic form $Q\left(x_{1}, \cdots, x_{n}\right)$ has $A^{*}(n)$ or $Q \in A^{*}(n)$, if (1) $Q$ is copositive and (2) if for all $i, j(i, j=1, \cdots, n)$ $Q-\varepsilon x_{i} x_{j}$ is not copositive for any $\varepsilon>0$. (That is, $Q$ is a copositive quadratic form which is "reduced" with respect to the extremes of $P)$.

We shall be concerned with the values taken by a quadratic form for $x_{i} \geqq 0(i=1, \cdots, n)$; to which end homogeneity usually permits us to restrict attention to those $x$ for which $x_{1}+\cdots+x_{n}=1$. Again following Diananda we call this $S(n)$, that is:

DeFinition 2.2. $S(n)=\left\{x\right.$ in $E_{n}: x_{1}+\cdots+x_{n}=1, x_{i} \geqq 0 \quad(i=$ $1, \cdots, n)\}$.

3. General results. We prove first a lemma which will be required later. It has an immediately corollary which serves to characterize the extreme copositive quadratic forms not belonging to $P+S$.

LemMA 3.1. If $Q=Q\left(x_{1}, \cdots, x_{n}\right)$ is a copositive quadratic form having a zero $u$ on $S(n)$, at which $u_{1}, \cdots, u_{m}>0, u_{m+1}=\cdots=u_{n}=0$ and $\partial Q(u) / \partial x_{i}=0(i=1, \cdots, n)$, then

$$
Q\left(x_{1}, \cdots, x_{n}\right)=L_{1}^{2}+\cdots+L_{s}^{2}+Q_{1}\left(x_{m+1}, \cdots, x_{n}\right)
$$

where $Q_{1}$ is copositive and by renumbering the $x_{i}(i=1, \cdots, m)$, we may assume that $L_{i}$ is a linear form in $x_{i}, \cdots, x_{n}(i=1, \cdots, s)$, $s<m$.

Proof. By Lemma 1 of Diananda $Q\left(x_{1}, \cdots, x_{m}, 0, \cdots, 0\right)$ is positive semi-definite and we collect squares with respect to $x_{1}, \cdots, x_{m}$ yielding 


$$
Q\left(x_{1}, \cdots, x_{m}, 0, \cdots, 0\right)=K_{1}^{2}+\cdots+K_{s}^{2}
$$

where by renumbering the $x_{i}(i=1, \cdots, m)$ we may assume that $K_{i}$ is a linear form in $x_{i}, \cdots, x_{m}(i=1, \cdots, s)$. Note that $Q(u)=0$ implies $s<m$. Thus we may express $Q\left(x_{1}, \cdots, x_{n}\right)$ as follows

$$
Q=L_{1}^{2}+\cdots+L_{s}^{2}+2 \sum_{i=s+1}^{m} \sum_{j=m+1}^{n} a_{i j} x_{i} x_{j}+Q_{1}\left(x_{m+1}, \cdots, x_{n}\right)
$$

where we may assume that $L_{i}$ is a linear form in $x_{i}, \cdots, x_{n}(i=1, \cdots, s)$. Now for any set of $x_{i} \geqq 0(i=s+1, \cdots, n)$ there exist values (possibly negative) for $x_{i}(i=1, \cdots, s)$ for which $L_{1}=\cdots=L_{s}=0$. Let $z$ be such a vector. Since $\partial Q(u) / \partial x_{i}=0(i=1, \cdots, n)$

$$
Q(z)=Q(z+k u)
$$

for all real $k$. But for $k>0$ sufficiently large $z+k u$ has all nonnegative components. Hence the copositivity of $Q$ together with equation 3.2 implies $Q(z) \geqq 0$. As $L_{1}(z)=\cdots=L_{s}(z)=0$, we see from (3.1) that

$$
Q_{2}\left(x_{s+1}, \cdots, x_{n}\right) \equiv 2 \sum_{i=s+1}^{m} \sum_{j=m+1}^{n} a_{i j} x_{i} x_{j}+Q_{1}\left(x_{m+1}, \cdots, x_{n}\right)
$$

is copositive in the variables $x_{s+1}, \cdots, x_{n}$ and hence (Lemma 2, Diananda) $a_{i j} \geqq 0(i=s+1, \cdots, m ; j=m+1, \cdots, n)$.

Let $m+1 \leqq j \leqq n$, then by $(3.1)$

$$
\partial Q(u) / \partial x_{j}=2 g_{1} L_{1}(u)+\cdots+2 g_{s} L_{s}(u)+2 \sum_{i=s+1}^{m} a_{i j} u_{i}+\partial Q_{1}(u) / \partial x_{j}=0
$$

where $g_{k}$ is the coefficient of $x_{j}$ in $L_{k}(k=1, \cdots, s)$. Now $Q(u)=0$ and $Q_{2}$ copositive imply by $(3.1)$ that $L_{k}(u)=0(k=1, \cdots, s)$; further $\partial Q_{1}(u) / \partial x_{j}=0$ as $u_{m+1}=\cdots=u_{n}=0$. Thus as $u_{i}>0 \quad(i=s+1$, $\cdots, m)$ we have $a_{i j}=0(i=s+1, \cdots, m ; j=m+1, \cdots, n)$. Hence

$$
Q=L_{1}^{2}+\cdots+L_{s}^{2}+Q_{1}\left(x_{m+1}, \cdots, x_{n}\right)
$$

where $Q_{1}$ is copositive and the $L_{i}$ 's are constituted as desired, which completes the proof.

Note that if $u_{1}, \cdots, u_{n-1}>0=u_{n}$ then $Q_{1}=a x_{n}^{2}$ and is copositive, hence $a \geqq 0$. Thus we have:

CoRollary 3.2. If $Q\left(x_{1}, \cdots, x_{n}\right)$ is a copositive quadratic form having a zero $u$ on $S(n)$, at which $u_{1}, \cdots, u_{n-1}>0=u_{n}$ and $\partial Q(u) / \partial x_{i}=$ $0(i=1, \cdots, n)$, then $Q$ is positive semi-definite.

CoRollary 3.3. If $Q\left(x_{1}, \cdots, x_{n}\right)$ is an extreme copositive quadratic form which has, among its zeros on $S(n)$, a zero u at which $\partial Q(u) / \partial x_{i}=$ 
$0(i=1, \cdots, n)$ then $Q$ is positive semi-definite.

Proof. By relabeling the variables we may assume that $u_{1}, \cdots$, $u_{m}>0$ and $u_{m+1}=\cdots=u_{n}=0$, where $1 \leqq m \leqq n$. Thus by the lemma

$$
Q\left(x_{1}, \cdots, x_{n}\right)=L_{1}^{2}+\cdots+L_{s}^{2}+Q_{1}\left(x_{m+1}, \cdots, x_{n}\right)
$$

with $Q_{1}$ copositive. Since $Q$ is extreme we must have $L_{2}^{2}+\cdots+L_{s}^{2}+$ $Q_{1}\left(x_{m+1}, \cdots, x_{n}\right) \equiv a L_{1}^{2}$ for some $a \geqq 0$, but this is impossible for $a>0$ (as $x_{1}$ appears in $L_{1}^{2}$ but not in $L_{2}^{2}+\cdots+L_{s}^{2}+Q_{1}$ ). Hence $a=0$ which implies that $Q$ is positive semi-definite.

Now every extreme copositive quadratic form in $n \geqq 2$ variables has zeros in $S(n)$, Lemma 4 of Diananda. Further if $u$ in $S(n)$ is a zero of a copositive quadratic form $Q$ then $\partial Q(u) / \partial x_{i} \geqq 0(i=1, \cdots, n)$, as one easily sees from copositivity. In fact $u_{i}>0$ implies $\partial Q(u) / \partial x_{i}=0$ and thus $\partial Q(u) / \partial x_{i}>0$ only if $u_{i}=0$. Thus the Corollary above allows us to conclude that every extreme copositive quadratic form not belonging to $P+S$ has at each of its zeros $u$ in $S(n)$ a component $u_{j}=0$, where $\partial Q(u) / \partial x_{j}>0$.

Our next theorem is the most important result of this paper; almost everything that is new here depends upon it in one way or another. In many respects it is related to Theorem 4.1 of Hall and Newman, [3].

THeOREM 3.4. A copositive quadratic form $Q\left(x_{1}, \cdots, x_{n}\right)$ has a zero $u$ on $S(n)$ with $u_{i}>0$ if and only if $Q-\varepsilon x_{i}^{2}$ is not copositive for any $\varepsilon>0,(1 \leqq i \leqq n)$.

Proof. If $Q$ has such a zero $u$ then $Q(u)-\varepsilon u_{i}^{2}=-\varepsilon u_{i}^{2}<0$ for any $\varepsilon>0$. On the other hand, proceeding by induction on $n$, let $n=1$, then $Q=q x_{1}^{2}(q \geqq 0)$, but $Q-\varepsilon x_{1}^{2}$ not copositive for all $\varepsilon>0$ implies that $q=0$, whence $Q$ has the zero $x_{1}=1$ of $S(1)$. For $n=2$, there are only 3 essentially distinct copositive $Q$ 's, these are (1) $q x_{1} x_{2}(q \geqq 0),(2) q_{11} x_{1}^{2}+2 q_{12} x_{1} x_{2}\left(q_{11}, q_{12}>0\right)$, (3) $q_{11} x_{1}^{2}+2 q_{12} x_{1} x_{2}+q_{22} x_{2}^{2}\left(q_{11}\right.$, $\left.q_{22}>0\right)$.

Case 1. $Q$ has zeros $x_{1}=1, x_{2}=0$ and $x_{1}=0, x_{2}=1$.

Case 2. $Q-\varepsilon x_{i}^{2}$ not copositive for all $\varepsilon>0$ implies that $i=2$, and $Q$ has the zero $x_{1}=0, x_{2}=1$ on $S(2)$.

Case 3. Since $q_{i i}>0$ we can assume without loss of generality that $q_{11}=q_{22}=1$, thus $Q=x_{1}^{2}+2 q_{12} x_{1} x_{2}+x_{2}^{2}=\left(x_{1}-x_{2}\right)^{2}+k x_{1} x_{2}$ where 
$k \geqq 0$ since $Q$ is copositive. Now $Q-\varepsilon x_{i}^{2}$ not copositive for all $\varepsilon>0$ implies (Lemma 4, Diananda [2] that $Q=\left(x_{1}-x_{2}\right)^{2}+k x_{1} x_{2}, k \geqq 0$ has a zero on $S(2)$ which can only be $x_{1}=x_{2}=1 / 2$, thus $k=0$. So $x_{1}=x_{2}=1 / 2$ is the desired zero on $S(2)$.

More generally, since $Q-\varepsilon x_{i}^{2}$ is not copositive for $\varepsilon>0$, there exists a nonnegative $n$-tuple $z$ [which may be assumed to be on $S(n)$ ] for which $Q(z)<\varepsilon z_{i}^{2}$. By taking successively smaller positive $\varepsilon$ 's we construct a sequence of nonnegative $n$-tuples, of which a subsequence converges to an $n$-tuple $u$ of $S(n)$. We denote the generic member of this subsequence by $v$, and note that continuity implies $Q(u)=0$. If $u_{i}>0$ we are done, so we assume $u_{i}=0$. Now relabel the variables so that $i=1$, and $u_{2}, \cdots, u_{m}>0$ and further $\partial Q(u) / \partial x_{j}=0$ for $j=$ $m+1, \cdots, k$ and $\partial Q(u) / \partial x_{j}>0(k+1 \leqq j \leqq n)$.

In the sequence of points $v=\left(v_{1}, \cdots, v_{n}\right)$ approaching $u=\left(0, u_{2}\right.$, $\left.\cdots, u_{m}, 0, \cdots, 0\right)$ put $v_{i}=u_{i}+w_{i}(i=1, \cdots, n)$ so that the $w_{i}$ all approach zero as $v$ approaches $u$. If $\partial Q(u) / \partial x_{n}=2 q_{n 1} u_{1}+\cdots+$ $2 q_{n n} u_{n}>0$ and $u_{n}=0$ we have

$$
\begin{aligned}
Q(v)= & Q\left(v_{1}, \cdots, v_{n-1}, 0\right) \\
& +w_{n}\left(\frac{\partial Q(u)}{\partial x_{n}}+2 q_{n 1} w_{1}+\cdots+2 q_{n n-1} w_{n-1}+q_{n n} w_{n}\right)
\end{aligned}
$$

Here if $v_{n}=w_{n}>0$, then for sufficiently small $w$ 's [since $\partial Q(u) / \partial x_{n}>0$ ] we have

$$
0 \leqq Q\left(v_{1}, \cdots, v_{n-1}, 0\right)<Q(v)<\varepsilon v_{1}^{2}
$$

Thus there exists a subsequence of the $v$ 's in which we may replace $v=\left(v_{1}, \cdots, v_{n}\right)$ by $v^{\prime}=\left(v_{1}, \cdots, v_{n-1}, 0\right)$ to yield a sequence which approaches $u$ and has the property $Q\left(v^{\prime}\right)<\varepsilon v_{1}^{2}$. Repeating this process we replace all the nonzero $v_{k+1}, \cdots, v_{n}$ in some suitable subsequence of the $v$ 's by zero. This yields a form $Q_{1}\left(x_{1}, \cdots, x_{k}\right)=Q\left(x_{1}, \cdots, x_{k}\right.$, $0, \cdots, 0$ ) and a sequence of $k$-tuples (in which we again designate the generic member by $v) v=\left(v_{1}, \cdots, v_{k}\right)$ approaching $u=\left(u_{1}, \cdots, u_{k}\right)$ for which $Q_{1}\left(v_{1}, \cdots, v_{k}\right)<\varepsilon v_{1}^{2}$ and for which $\partial Q_{1}(u) / \partial x_{i}=0$ for $i=$ $2, \cdots, k$ (copositivity implies $\partial Q_{1}(u) / \partial x_{i}=0$ if $\left.u_{i}>0\right)$. Since $u_{1}=0$ we have

$$
\begin{aligned}
Q_{1}\left(v_{1}, \cdots, v_{k}\right)= & w_{1}\left(q_{11} w_{1}+\frac{\partial Q_{1}(u)}{\partial x_{1}}+2 q_{12} w_{2}+\cdots+2 q_{1 k} w_{k}\right) \\
& +Q_{1}\left(0, v_{2}, \cdots, v_{k}\right)
\end{aligned}
$$

with $w_{1}=v_{1}>0$ (follows from 3.3). Since $Q_{1}\left(0, v_{2}, \cdots, v_{k}\right) \geqq 0$ the inequality $Q_{1}\left(v_{1}, \cdots, v_{k}\right)<\varepsilon v_{1}^{2}$ can only hold for sufficiently small $w$ 's if $\partial Q_{1}(u) / \partial x_{1}=0$. Hence for $Q_{1}\left(x_{1}, \cdots, x_{k}\right)$ we have $Q_{1}(u)=0$ and 
$\partial Q_{1}(u) / \partial x_{i}=0(i=1, \cdots, k)$.

Applying Lemma 3.1 we get

$$
Q_{1}\left(x_{1}, \cdots, x_{k}\right)=L_{2}^{2}+\cdots+L_{s}^{2}+Q_{2}\left(x_{1}, x_{m+1}, \cdots, x_{k}\right)
$$

with $Q_{2}$ copositive and we may assume that $L_{i}$ is a linear form in $x_{1}, x_{i}, \cdots, x_{k}(i=2, \cdots, s)$.

If $\left(v_{1}, \cdots, v_{k}\right)=v$ is one of our $k$-tuples for which $Q_{1}(v)<\varepsilon v_{1}^{2}$ then (3.4) shows that $Q_{2}\left(v_{1}, v_{m+1}, \cdots, v_{k}\right)<\varepsilon v_{1}^{2}$. If $Q_{2}$ does not contain $x_{1}^{2}$ then $Q_{2}(1,0, \cdots, 0)=0$; whereas if $x_{1}^{2}$ appears in $Q_{2}$ the induction hypothesis applies as $0<k-m+1<n$. So in either case $Q_{2}$ has a zero $z=\left(z_{1}, z_{m+1}, \cdots, z_{k}\right)$ on $S(k-m+1)$ with $z_{1} \neq 0$. Now $Q_{1}(u)=0$, hence (by 3.4) $L_{2}(u)=\cdots=L_{s}(u)=0$ where $u_{1}=0=u_{m+1}=\cdots=u_{k}$ and $u_{i}>0(i=2, \cdots, m)$. Due to the structure of the $L_{i}^{\prime}$ 's, the equations

$$
\begin{aligned}
& L_{2}=\cdots=L_{s}=x_{s+1}=\cdots=x_{m}=0, \\
& x_{1}=z_{1}, x_{m+1}=z_{m+1}, \cdots, x_{k}=z_{k}
\end{aligned}
$$

have a unique solution $\left(z_{1}, \cdots, z_{k}\right)$ where the $z_{i}(i=2, \cdots, s)$ may be negative. Taking $t>0$ large enough we can insure that $w_{i} \equiv t u_{i}+$ $z_{i} \geqq 0 \quad(i=1, \cdots, k)$ and $w_{i}>0$ for $(i=1, \cdots, m)$. Since $w_{1}=z_{1}$ and $w_{i}=z_{i}(i>m)$ we have $Q_{2}(w)=0$; further $L_{2}(w)=\cdots=L_{s}(w)=$ 0 . Hence (3.4) implies that $Q_{1}(w)=0$ with $w_{1}>0$, thus there is a zero of $Q_{1}$ on $S(k)$ with $x_{1}>0$. Since $Q_{1}\left(x_{1}, \cdots, x_{k}\right)=Q\left(x_{1}, \cdots, x_{k}\right.$, $0, \cdots, 0)$ the theorem is proved.

If we apply this result to forms having $A^{*}(n)$ we get

CoRollary 3.5. If $Q\left(x_{1}, \cdots, x_{n}\right)$ is a quadratic form having $A^{*}(n)$ then for every index $i(1 \leqq i \leqq n), Q$ has a zero $u$ on $S(n)$ with $u_{i}>0$.

In stating the associated result for extreme forms we must be careful to exclude extremes of the type $a x_{i}^{2}, a>0$, hence we limit ourselves to the case where $n \geqq 2$.

CoROLlaRY 3.6. If $Q\left(x_{1}, \cdots, x_{n}\right), n \geqq 2$, is an extreme copositive quadratic form then for every index $i(1 \leqq i \leqq n), Q$ has a zero $u$ on $S(n)$ with $u_{i}>0$.

Another immediate consequence of Theorem 3.4 is:

COROLlaRY 3.7. If $Q\left(x_{1}, \cdots, x_{n}\right)$ is a quadratic form having $A^{*}(n)$, and if, among its zeros on $S(n), Q$ has a zero $u$ with

$$
u_{1}, \cdots, u_{k-1}, u_{k+1}, \cdots, u_{n}>0=u_{k}
$$


then $Q$ is positive semi-definite.

Proof. By Lemma 11, Diananda [2] we are done if we can show that $Q$ has a zero with $u_{k}>0$, but this follows from Corollary 3.5.

As extreme forms in $n \geqq 3$ variables have $A^{*}(n)$ Corollary 3.7 is immediately applicable to them. Our final result of this section allows us to construct extreme copositive forms in $n$ variables from those in $n^{\prime}$ variables, for $2<n^{\prime}<n$.

THEOREM 3.8. If $Q_{n}$ is an extreme copositive quadratic form in $n \geqq 3$ variables $x_{1}, \cdots, x_{n}$ then replacing any $x_{i}$ by $x_{i}+x_{n+1}$ in $Q_{n}$ yields a new copositive form $Q_{n+1}$, which is extreme.

Proof. We may assume that $i=n$ and that $q_{j j}=1$ for all $j$ (since $n \geqq 3$ and $Q_{n}$ is extreme, see Lemma 2, Diananda). $Q_{n+1}$ is obviously copositive. Now suppose that

$$
Q_{n+1}=Q^{\prime}+Q^{\prime \prime}
$$

with $Q^{\prime}, Q^{\prime \prime}$ copositive, then by setting $x_{n+1}=0$ and $x_{n}=0$ in turn, we get

$$
Q_{n}=a Q_{n}+(1-a) Q_{n}, \quad Q_{n}^{*}=b Q_{n}^{*}+(1-b) Q_{n}^{*} \quad(0 \leqq a, b \leqq 1)
$$

by the extremity of $Q_{n}, Q_{n}^{*}$. By (3.5) the coefficients of $x_{11}^{2}$ in $a Q_{n}$ and $b Q_{n}^{*}$ are the same and as $q_{11}=1 \neq 0$ we have $a=b$. So we see that

$$
\begin{aligned}
& Q^{\prime}=a\left(Q_{n}+x_{n+1}^{2}+2 \sum_{i=1}^{n-1} q_{i n} x_{i} x_{n+1}\right)+k x_{n} x_{n+1} \\
& Q^{\prime \prime}=(1-a)\left(Q_{n}+x_{n+1}^{2}+2 \sum_{i=1}^{n-1} q_{i n} x_{i} x_{n+1}\right)+t x_{n} x_{n+1}
\end{aligned}
$$

with $k+t=2$. Thus

$$
\begin{aligned}
Q^{\prime} & =a Q_{n+1}+(k-2 a) x_{n} x_{n+1}, \\
Q^{\prime \prime} & =(1-a) Q_{n+1}+(t+2 a-2) x_{n} x_{n+1}
\end{aligned}
$$

Since $Q_{n}$ is extreme it has a zero $u$ in $S(n)$ with $u_{n}>0$ (Corollary $3.6)$, thus $\left(u_{1}, \cdots, u_{n-1}, u_{n} / 2, u_{n} / 2\right)$ is a zero of $Q_{n+1}$ in $S(n+1)$ with $x_{n} x_{n+1}>0$. At this zero $4 Q^{\prime}=(k-2 a) u_{n}^{2}$ and $4 Q^{\prime \prime}=(t+2 a-$ 2) $u_{n}^{2}$, thus the copositivity of $Q^{\prime}, Q^{\prime \prime}$ insures that $(k-2 a) \geqq 0$ and $(t+2 a-2) \geqq 0$ respectively. But $Q_{n+1}=0$ here, so $k-2 a=t+$ $2 a-2=0$. Thus $Q^{\prime}=a Q_{n+1}, Q^{\prime \prime}=(1-a) Q_{n+1}$ and so $Q_{n+1}$ is extreme.

The author is indebted to Professor M. Hall, Jr. for many enlightening discussions during the preparation of this paper. 


\section{REFERENCES}

1. L. D. Baumert, Extreme copositive quadratic forms, Dissertation, California Institute of Technology, 1965.

2. P. H. Diananda, On non-negative forms in real variables some or all of which are non-negative, Proc. Cambridge Philos. Soc. 58 (1962), 17-25.

3. M. Hall, Jr. and M. Newman, Copositive and completely positive quadratic forms, Proc. Cambridge Philos. Soc. 59 (1963), 329-339.

4. T. S. Motzkin, Copositive quadratic forms, National Bureau of Standards Report 1818 (1952), 11-12.

5. - Quadratic forms positive for non-negative variables not all zero, Notices of Amer. Math. Soc. 12 (1965), 224.

Received April 5, 1965. This research was supported in part by the Jet Propulsion Laboratory, California Institute of Technology, under Contract No. NAS 7-100 with the National Aeronautics and Space Administration. 


\title{
PACIFIC JOURNAL OF MATHEMATICS
}

\author{
EDITORS
}

\section{H. SAMELSON}

Stanford University

Stanford, California

J. P. JANS

University of Washington

Seattle, Washington 98105
J. DugundJI

University of Southern California

Los Angeles, California 90007

RICHARD ARENS

University of California

Los Angeles, California 90024

\section{ASSOCIATE EDITORS}

\section{E. F. BECKENBACH}

B. H. NEUMANN

F. WOLF

K. YosidA

\section{SUPPORTING INSTITUTIONS}

\author{
UNIVERSITY OF BRITISH COLUMBIA \\ CALIFORNIA INSTITUTE OF TECHNOLOGY \\ UNIVERSITY OF CALIFORNIA \\ MONTANA STATE UNIVERSITY \\ UNIVERSITY OF NEVADA \\ NEW MEXICO STATE UNIVERSITY \\ OREGON STATE UNIVERSITY \\ UNIVERSITY OF OREGON \\ OSAKA UNIVERSITY \\ UNIVERSITY OF SOUTHERN CALIFORNIA
}

\author{
STANFORD UNIVERSITY \\ UNIVERSITY OF TOKYO \\ UNIVERSITY OF UTAH \\ WASHINGTON STATE UNIVERSITY \\ UNIVERSITY OF WASHINGTON \\ AMERICAN MATHEMATICAL SOCIETY \\ CHEVRON RESEARCH CORPORATION \\ TRW SYSTEMS \\ NAVAL ORDNANCE TEST STATION
}

Mathematical papers intended for publication in the Pacific Journal of Mathematics should be typewritten (double spaced). The first paragraph or two must be capable of being used separately as a synopsis of the entire paper. It should not contain references to the bibliography. Manuscripts may be sent to any one of the four editors. All other communications to the editors should be addressed to the managing editor, Richard Arens at the University of California, Los Angeles, California 90024.

50 reprints per author of each article are furnished free of charge; additional copies may be obtained at cost in multiples of 50 .

The Pacific Journal of Mathematics is published monthly. Effective with Volume 16 the price per volume (3 numbers) is $\$ 8.00$; single issues, $\$ 3.00$. Special price for current issues to individual faculty members of supporting institutions and to individual members of the American Mathematical Society: $\$ 4.00$ per volume; single issues $\$ 1.50$. Back numbers are available.

Subscriptions, orders for back numbers, and changes of address should be sent to Pacific Journal of Mathematics, 103 Highland Boulevard, Berkeley 8, California.

Printed at Kokusai Bunken Insatsusha (International Academic Printing Co., Ltd.), No. 6, 2-chome, Fujimi-cho, Chiyoda-ku, Tokyo, Japan.

PUBLISHED BY PACIFIC JOURNAL OF MATHEMATICS, A NON-PROFIT CORPORATION

The Supporting Institutions listed above contribute to the cost of publication of this Journal, but they are not owners or publishers and have no responsibility for its content or policies. 


\section{Pacific Journal of Mathematics \\ Vol. 19, No. $2 \quad$ June, 1966}

Leonard Daniel Baumert, Extreme copositive quadratic forms . ......... 197

Fred James Bellar, Jr., Pointwise bounds for the second initial-boundary value problem of parabolic type ........................ 205

L. Carlitz and David Paul Roselle, Restricted bipartite partitions ........ 221

Robin Ward Chaney, On the transformation of integrals in measure space ........................................... 229

Colin W. Clark, An embedding theorem for function spaces ........... 243

Edwin Duda, A theorem on one-to-one mappings................ 253

Ben Fitzpatrick, Jr. and Donald Reginald Traylor, Two theorems on metrizability of Moore spaces.......................... 259

Allen Roy Freedman, An inequality for the density of the sum of sets of vectors in $n$-dimensional space ....................... 265

Michael Friedberg, On representations of certain semigroups .......... 269

Robert William Gilmer, Jr., The pseudo-radical of a commutative ring . . . . 275

Hikosaburo Komatsu, Fractional powers of operators ............... 285

Daniel Rider, Transformations of Fourier coefficients ................. 347

David Alan Sánchez, Some existence theorems in the calculus of variations ...................................... 357

Howard Joseph Wilcox, Pseudocompact groups............... 365

William P. Ziemer, Some lower bounds for Lebesgue area ............. 381 